\title{
Blueberry Extracts Protect Testis from Hypobaric Hypoxia Induced Oxidative Stress in Rats
}

\author{
Andrea Zepeda, ${ }^{1}$ Luis G. Aguayo, ${ }^{2}$ Jorge Fuentealba, ${ }^{2}$ Carolina Figueroa, ${ }^{1}$ \\ Alejandro Acevedo, ${ }^{1}$ Perla Salgado, ${ }^{1}$ Gloria M. Calaf,, ${ }^{3,4}$ and Jorge Farías ${ }^{1}$ \\ ${ }^{1}$ Departamento de Ingeniería Química, Facultad de Ingeniería, Ciencias y Administración, Universidad de La Frontera, Temuco, Chile \\ ${ }^{2}$ Neuroactive Drugs Screening Unit, Departamento de Fisiología, Facultad de Ciencias Biológicas, Universidad de Concepción, \\ Concepción, Chile \\ ${ }^{3}$ Instituto de Alta Investigación, Universidad de Tarapacá, Arica, Chile \\ ${ }^{4}$ Center for Radiological Research, Columbia University Medical Center, New York, NY, USA
}

Correspondence should be addressed to Jorge Farías, jfarias@ufro.cl

Received 4 June 2012; Revised 22 August 2012; Accepted 26 September 2012

Academic Editor: Vincent Pialoux

Copyright (c) 2012 Andrea Zepeda et al. This is an open access article distributed under the Creative Commons Attribution License, which permits unrestricted use, distribution, and reproduction in any medium, provided the original work is properly cited.

\begin{abstract}
Exposure to hypobaric hypoxia causes oxidative damage to male rat reproductive function. The aim of this study was to evaluate the protective effect of a blueberry extract (BB-4) in testis of rats exposed to hypobaric hypoxia. Morphometric analysis, cellular DNA fragmentation, glutathione reductase (GR), and superoxide dismutase (SOD) activities were evaluated. Our results showed that supplementation of BB-4 reduced lipid peroxidation, decreased apoptosis, and increased GR and SOD activities in rat testis under hypobaric hypoxia conditions $(P<0.05)$. Therefore, this study demonstrates that blueberry extract significantly reduced the harmful effects of oxidative stress caused by hypobaric hypoxia in rat testis by affecting glutathione reductase and superoxide dismutase activities.
\end{abstract}

\section{Introduction}

Berries are a recognized source of antioxidants since they contain phytochemicals, nonenzymatic factors of plant origin that significantly benefit health $[1,2]$. Such extracts have proven to be effective in preventing the effects of oxidative stress under different pathological conditions [3-6]. Among the different species, there is a group classified as blueberries that have a dark color due to anthocyanins and polyphenols as principal pigments with antioxidant activities [3]. Phytochemicals have been demonstrated to be powerful inhibitors of lipid peroxidation when compared to other classic antioxidants $[3,7]$, and the protective effect of polyphenols against oxidative damage seems to be via glutathione system [8].

The enzymatic mechanism against oxidative stress is made of free radical scavengers like superoxide dismutase (SOD), catalase (CAT), and the glutathione-dependent enzymes such as glutathione peroxidase (GPx), glutathione S-transferase (GSH), and Glutathione reductase (GR) [9].
GR and enzymatic antioxidant mechanisms play an essential role in preventing oxidative damage in cells and tissues [10].

We have previously described that hypobaric hypoxia induced oxidative damage, decreased glutathione reductase activity and ascorbic acid, and had a protective role against oxidative stress [11]. The effect of a reduced spermatogenesis under hypobaric hypoxia [12] is accompanied by an increased vascularization and reactive oxygen species (ROS) in the testis $[13,14]$. These vascular changes are induced by ROS via inhibition of prolyl hydroxylase domain (PHD) proteins [11]. The activity of PHD seems to be restored by a supplement of ascorbic acid [15] making it possible to generate strategies for administering antioxidants to prevent the effects of hypobaric hypoxia as previously suggested $[14,16,17]$.

Previously, It has been demonstrated that enriched blueberries reduced the adverse effects of oxidative stress in rat neuron cell lines and brain tissues $[18,19]$. Such extract has shown to cross the blood-brain barrier $[19,20]$. Brain 
homeostasis and spermatogenesis depend on blood-to-brain and blood-to-germ cells transport of metabolites and substances [21], therefore it was of interest to determine whether the protective effect can be induced in rat testis in vivo model. The aim of this work was to evaluate the protective effect of a blueberry-enriched polyphenol extract (BB-4) against oxidative stress in rat testis exposed to hypobaric hypoxia.

\section{Materials and Methods}

2.1. Experimental Design. Ten-week-old Sprague Dawley rats (Rattus norvegicus specie) were divided into six groups (5 rats per group): (1) normobaric conditions (Nx); (2) Nx plus administration of physiological solution (PS); (3) $\mathrm{Nx}$ plus blueberry extract (BB-4), rich in polyphenols, (BB-4); (4) hypobaric hypoxia (HH); (5) $\mathrm{HH}$ plus PS and (6) $\mathrm{HH}$ + BB-4. Rats were housed under a 12 hours of Light: 12 hours of Dark cycles and the humidity was $61 \pm 9 \%$. BB-4 was administered intraperitoneally $(10 \mathrm{mg}$ dry extract $/ \mathrm{kg}$ of body weight) or with physiological solution $(1 \mathrm{~mL}$ of $\mathrm{NaCl}$ $9 \mathrm{mg} / \mathrm{mL}$ ) at 96 -hour intervals. The selection of the dose and route of administration of BB-4 was based on previous work reported $[11,14]$ where the protective effect of compounds did not affect the liver as analyzed by the presence of transaminases in the blood of animals. Groups 4, 5, and 6 were exposed to $\mathrm{HH}$ conditions for 96 hours in a hypobaric chamber ( 428 tor; pO2: $89.6 \mathrm{mmHg}$ ) for a period of 32 days. The desired pressure inside the hypobaric chamber was achieved by pressure changes simulating altitude increases of 150 meters per minute. The animals in the Nx groups were lodged in the same room as the $\mathrm{HH}\left(22^{\circ} \mathrm{C}, 15 \mathrm{~g}\right.$ of pellet meals per day and $250 \mathrm{~mL}$ of water per rat). All procedures complied with the principles of animal care outlined by the National Society Laboratory and the Medical Research, and the Guide for the Care and Use of Laboratory Animals (Institute of Animal Laboratory Resources, 1996).

2.2. Blueberry Extracts. Six polyphenol-enriched blueberry extracts were obtained from fresh blueberries, locally harvested, using different solvents (with increasing polarity), and denominated BB-1-6, depending on the solvents employed (chloroform, acetone/water, ethanol, ethanol/water, water/acetone, or methanol). The most active extract was obtained with ethanol/water and was denominated BB-4. To obtain the enriched polyphenol extracts (BB), we used an Amberlite XAD-7 adsorber resin (Merck, Darmstadt, Germany) and an Amberlite XAD-2 adsorber resin (Supelpack 2; Sigma-Aldrich). The extract obtained had $1.5 \%$ polyphenols for each $100 \mathrm{~g}$ of fresh fruit, where the main components were rutin $(0.34 \%)$ and isoquercetin $(0.42 \%)$. The BB-4 extract was shown to be the most active in the preliminary activity screening test and it was then characterized to determine its polyphenol composition through HPLC techniques. The final BB-4 extract was dried and diluted in dimethylsulfoxide (DMSO) to a final concentration of
$810 \mathrm{mg} /$ liter. Different dilutions from $1: 10$ to $1: 100,000$ were prepared daily in external solution containing $5 \mathrm{mM}$ $\mathrm{CaCl}_{2}, 100 \mathrm{mM} \mathrm{NaCl}, 45 \mathrm{mM}$ tetraethylammonium chloride (TEACl), $10 \mathrm{mM}$ HEPES, $5.5 \mathrm{mM} \mathrm{KCl}$, and $10 \mathrm{mM}$ glucose [18].

2.3. Organ Collection. The animals were weighed and sacrificed by cervical dislocation. The testes were removed and placed in a saline buffer PBS $(137 \mathrm{mM} \mathrm{NaCl}, 2.7 \mathrm{mM} \mathrm{KCl}$, $10 \mathrm{mM}$ sodium phosphate dibasic, $2 \mathrm{mM}$ sodium phosphate monobasic, pH 7.2) (Sigma Chemicals, St Louis, MO, USA) for further studies.

2.4. Preparation of Tissue Homogenate and Protein Assay. The $100 \%$ of testis were completely homogenized in $0.5 \mathrm{~mL}$ of extraction buffer ( $50 \mathrm{mM}$ Tris, $100 \mathrm{mM} \mathrm{NaCl}, 1 \mathrm{mM}$ EDTA, $2.5 \mathrm{mM}$ EGTA, Tween-20 0.1\% v/v pH 7.4, $100 \mu \mathrm{g} / \mathrm{mL}$ PMSF (Sigma Chemicals, St Louis, MO, USA) with a Potter glass homogenizer (Glass-Col K4424, CA, USA) at $50 \mathrm{rpm}$. Then the samples were centrifuged for 30 minutes at $4^{\circ} \mathrm{C}$. The protein concentration was determined on the supernatant using the Coomassie blue method [22].

2.5. Glutathione Reductase Expression Determined by Western Blot (SDS/PAGE). Aliquots of tissue homogenate of testis containing equal concentration of proteins, $50 \mu \mathrm{g}$, were electrophoresed $(120 \mathrm{mV})$ in a $12 \%$ SDS/PAGE gel as previously described by Farias et al. [11], using a primary anti-rat glutathione reductase antibody against rabbit (1:500 dilution) (Santa Cruz Biotechnology, Santa Cruz, CA, USA) and a secondary anti-rabbit antibody (Jackson Immune Research Laboratories, PA, USA). $\beta$ tubulin was used as a loading control in all Western blot assays. The bands obtained were analyzed with Image J Software (http://rsbweb.nih.gov/ij/download.html) and the integrated density values of the glutathione reductase bands were normalized by dividing by the value of the loading control band.

2.6. Histological Procedures. Testis from each animal was weighed and fixed in $4 \%$ paraformaldehyde in $0.1 \mathrm{M}$ phosphate buffer, $\mathrm{pH} 7.2$, for 24 hours at room temperature. The testicles were embedded in paraffin after dehydration in ascending alcohol concentrations. Five sections were cut from the equatorial zone toward the testicular apex and mounted on glass slides and stained with hematoxylin-eosin sections.

2.7. Determination of Cellular DNA Fragmentation. In order to detect apoptosis by DNA fragmentation, an in situ Oligo Ligation method (ApoTag ISOL, Q-BIOgene, UK) was carried out as described by Lesauskaite et al. [23]. This method is based upon the specificity of the enzyme T4 DNA ligase [24]. In these experiments, we utilized five $5 \mu \mathrm{m}$ tissue sections that were obtained from rat testicles from the equatorial zone toward the testicular apex. The distance between the sections corresponded to $120 \mu \mathrm{m}$. This procedure was repeated for every condition tested. 
2.8. Determination of Lipid Peroxidation in the Testis. The protocol for the thiobarbituric acid (TBA) (Sigma Chemicals, St Louis, MO, USA) assay was carried out as described by Draper and Hadley [25]. $5 \mathrm{mg}$ of tissue homogenates were centrifuged at $10000 \mathrm{~g}$ for $10 \mathrm{~min}$ at $4^{\circ} \mathrm{C}$ to sediment mitochondria and cell debris. The sediments were suspended in PBS $\mathrm{pH} 7.4$, mixed with BHT-TCA solution $(1 \% \mathrm{w} / \mathrm{v}$ BHT dissolved in 20\% w/v TCA), and centrifuged at $1000 \mathrm{~g}$ for $5 \mathrm{~min}$. Supernatant was then mixed with $0.5 \mathrm{~N} \mathrm{HCl}$ and $120 \mathrm{mM}$ TBA in $26 \mathrm{mM}$ Tris and heated in a water bath at $80^{\circ} \mathrm{C}$ for $10 \mathrm{~min}$. After cooling, the absorbance of the resulting chromophore was determined at $532 \mathrm{~nm}$ using a OPTIZEN UV-Visible spectrophotometer (3220UV) and MDA production was determined by using an extinction coefficient of $1.56 \times 10^{5} \mathrm{M}^{-1} \mathrm{~cm}^{-1}$. The results were expressed as nmol of MDA equivalents/mg tissue.

2.9. Determination of Glutathione Reductase Activity in the Testis. To measure glutathione reductase activity, homogenates were thawed at room temperature and centrifuged at $700 \times \mathrm{g}$ for $10 \mathrm{~min}$, after which $20 \mu \mathrm{l}$ of supernatant was added to quartz cuvettes containing a fresh solution of $0.44 \mathrm{mM}$ GSSG, $0.30 \mathrm{M}$ EDTA, in $0.1 \mathrm{M}$ phosphate buffer- $\mathrm{pH} \quad 7.0$-and $0.036 \mathrm{MNADPH}$ was added just before the enzymatic determination as the starting reagent. The assay was run at $340 \mathrm{~nm}$ for $4 \mathrm{~min}$ with absorbance readings taken every $30 \mathrm{~s}$. Glutathione reductase activity was estimated using NADPH extinction coefficient of $6.2 \mathrm{mM}^{-1} \cdot \mathrm{cm}^{-1}$ and expressed as $\mathrm{U} / \mathrm{mg}$ of protein $[11,26]$.

\subsection{Determination of Superoxide Dismutase Activity in} the Testis. Superoxide dismutase activity was measured by the autoxidation of pyrogallol method as described by $\mathrm{S}$. Marklund and G. Marklund [27]. The activity of superoxide dismutase was assayed in $1000 \times \mathrm{g}$ supernatants of testis homogenates. One unit of superoxide dismutase was defined as the amount of protein that caused 50\% pyrogallol autoxidation inhibition. Superoxide dismutase activity was expressed as $\mathrm{U} / \mathrm{mg}$ protein.

2.11. Evaluation of Hematocrit, Body Weight, and Testicular Mass Relative to Body Weight. Blood samples were obtained with a needle from the left ventricle of the rat once sacrificed. The percentage of hematocrit was determined by centrifugation of the capillary tube with heparinized blood in a microhematocrit centrifuge (IEC Model MB, GSR Technical Sales, Canada). The animals were weighed and the testicular mass relative to body weight was determinate at the end of 32 days: (Testicular mass/body weight) $* 100=(\%)$.

2.12. Statistical Analysis. The results were analyzed as previously described by Farias et al. [11] by the two-way ANOVA in order to determine the presence of a significant interaction between the environmental factors (sea level and high altitude) and the injection factors (without injection, vehicle and dose), making it possible to determine whether the treatment with BB-4 under $\mathrm{IHH}$ produces any significant effect on the variables different to that produced in $\mathrm{Nx}$.
Also, the two-way ANOVA enabled to determine whether at least one level of each factor (environmental or injection) affected the results and to determine whether the changes in the barometric pressure or the administration of BB-4 produced any significant effect on the results. The statistical significance was established to $P<0.05$ for all analyses and a Bonferroni test was performed to compare treatments. Data were analyzed using the Graph Pad Prism Software v4.0 (San Diego, CA, USA). The results are presented in graphs with standard deviation of the mean (SD).

\section{Results}

The effect of hypobaric hypoxia exposure on testicular mass, testicular mass relative to body weight, diameter of seminiferous tubule, and height of epithelium was reversed with treatment with BB-4 $(P<0.05)$. Indeed, all these parameters came back to similar levels to those obtained in Nx (Figures 1(a), 1(b), and 1(c); Table 1). The hypoxia hypobaric condition induced apoptotic DNA fragmentation in spermatogenic cells in rats (Figure 1(d); $P<0.05$ ). However, in rats subjected to hypobaric hypoxia and treated with BB-4, the apoptotic index significantly decreased $(P<$ 0.05). On the other hand, lipid peroxidation (TBARS) was significantly higher $(P<0.05)$ under hypobaric hypoxia as compared to normoxic conditions in the testis as shown in Figure 2(a). The blueberry extract (BB-4) did not affect rats exposed under normoxia; however, this substance reduced lipid peroxidation in treated rats with the extract $(P<0.05)$. BB-4 seemed to protect the testis only under hypobaric hypoxic conditions.

There was a significant increase in glutathione reductase and superoxide dismutase activities under hypobaric hypoxia in comparison to normoxic groups $(P<0.05)$ as shown in Figures 2(b) and 2(c). BB-4 seemed to protect the testis under hypobaric hypoxic conditions when the enzymes SOD and GR increased.The specific activities of these enzymes significantly increased $(P<0.05)$ in rats treated with blueberry extract and subjected to hypobaric hypoxia $(P<0.05)$. There were no significant differences in glutathione reductase protein expression under hypoxia in comparison to normoxic groups $(P>0.05)$, as observed in Figure 2(d). The hematocrit was significantly greater $(P<$ $0.05)$ in groups subjected to hypobaric conditions when compared to normoxic groups (Figure 3(b)). The blueberry extract did not have any effect on the polycythemia that usually characterizes exposure to hypobaric hypoxia as a compensatory mechanism to the drop in arterial pressure of oxygen. Our results showed a significant decrease $(P<$ 0.05 ) in body weight of hypobaric hypoxia groups in comparison to normoxic groups (Figure 3(a)). However, blueberry extract treatment did not affect this parameter under any environmental conditions.

\section{Discussion}

These results showed that the levels of lipid peroxidation in all groups subjected to hypobaric hypoxia induced 


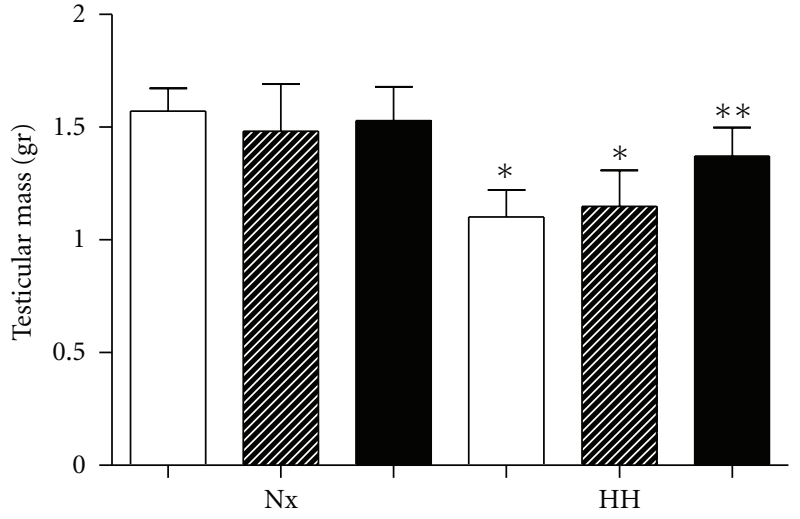

(a)

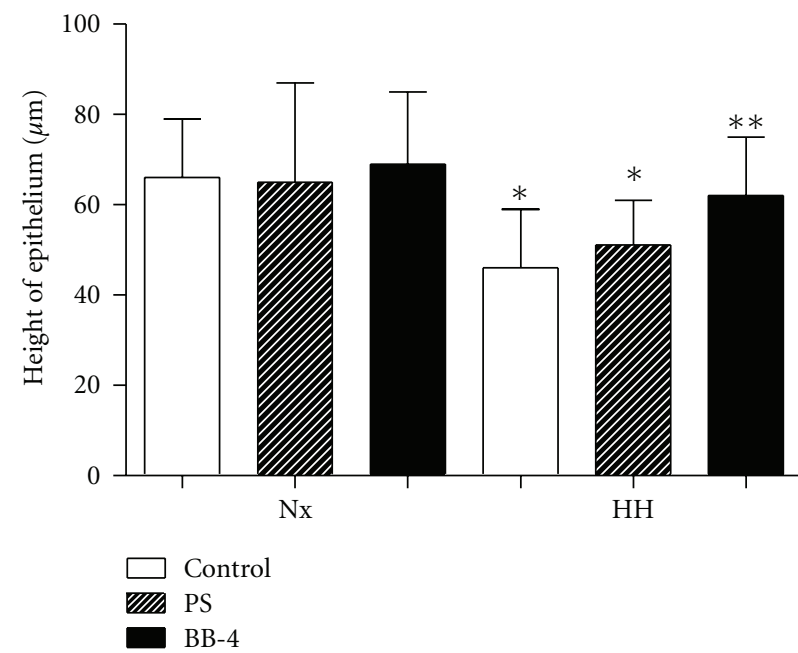

(c)

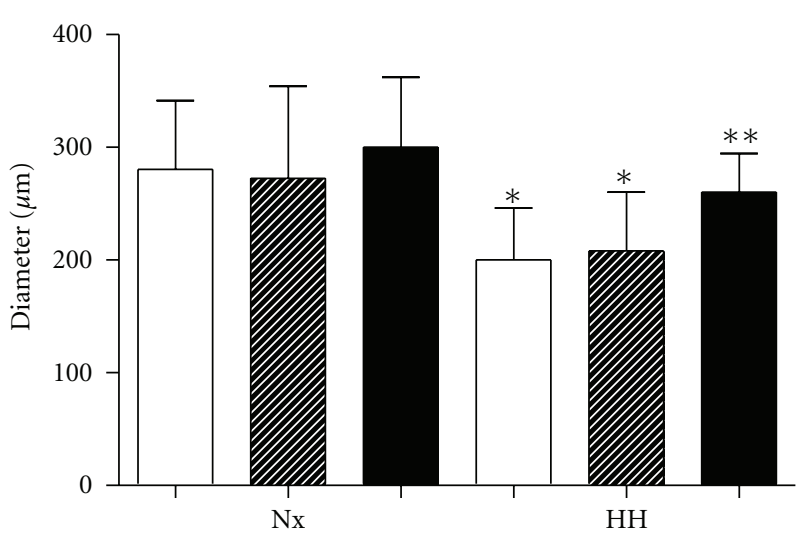

(b)

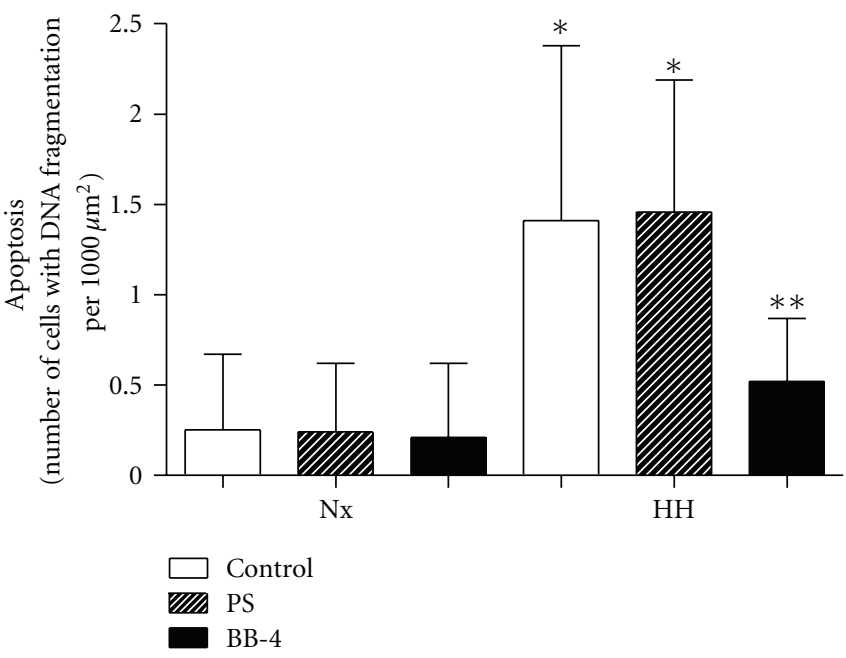

(d)

FIGURE 1: Effect of intermittent hypobaric hypoxia and BB-4 on (a) testicular mass (gr), (b) diameter ( $\mu$ m), epithelium height, (c) and (d) apoptotic index (number of cells with fragmented DNA per $1000 \mu \mathrm{m}^{2}$ ). Rats were submitted to intermittent hypobaric hypoxia (IHH) or normobaric $(\mathrm{Nx})$ conditions, with or without treatment of blueberry extract (BB-4). PS: rats treated with physiological NaCl solution. Experiment: 32 -day treatment. Bars indicate the mean $\pm \mathrm{SD}(n=5) .{ }^{*} P<0.05$ (HH versus Nx control); ${ }^{* *} P<0.05(\mathrm{HH}+\mathrm{BB}-4$ versus $\mathrm{HH}$ control).

TABLE 1: Testicular mass relative to body weight (\%).

\begin{tabular}{lccccc}
\hline $\mathrm{Nx}$ & $\mathrm{Nx}+\mathrm{PS}$ & $\mathrm{Nx}+\mathrm{BB}-4$ & $\mathrm{HH}$ & $\mathrm{HH}+\mathrm{PS}$ & $\mathrm{HH}+\mathrm{BB}-4$ \\
\hline $0.44 \pm 0.02$ & $0.41 \pm 0.03$ & $0.43 \pm 0.02$ & $0.40 \pm 0.01^{*}$ & $0.40 \pm 0.04^{*}$ & $0.48 \pm 0.03^{* *}$ \\
\hline
\end{tabular}

Rats were submitted to intermittent hypobaric hypoxia (IHH) or normobaric (Nx) conditions, with or without treatment of blueberry extract (BB-4). PS: rats treated with physiological $\mathrm{NaCl}$ solution. (Testicular mass/body weight) $* 100=(\%)$. Mean $\pm \mathrm{SD} . * P<0.05(\mathrm{HH}$ versus $\mathrm{Nx}$ control); $* * P<0.05(\mathrm{HH}+$ BB-4 versus $\mathrm{HH}$ control).

oxidative stress causing a decrease in testicular mass, arrest of spermatogenesis, and an increase in apoptosis. In previous studies, we reported that hypobaric hypoxia caused oxidative damage and histological changes in testis [11-13].

The production of reactive oxygen species at the testicular level can be especially accentuated by the presence of a basal hypoxic microenvironment and abundance of polyunsaturated fatty acids $[28,29]$. On the other hand, the activities of glutathione reductase and superoxide dismutase were significantly reduced. We previously found that enzyme expression did not change in rat testis under hypobaric hypoxia [11].

Several studies have demonstrated the beneficial effects of blueberries against oxidative stress. Blueberries have the ability to neutralize oxygen-containing free radicals [30]. Phenolic compounds with strong antioxidant properties are found in the diet and can prevent oxidative damage as a result of their ability to scavenge reactive oxygen species [31]. It has been reported that blueberries have protective effects against oxidative damage in animals $[19,32]$. Animals exposed to 


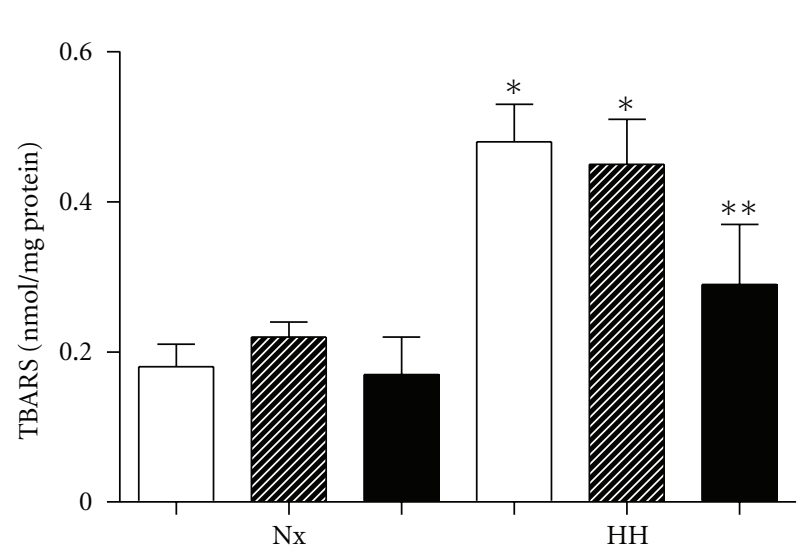

(a)

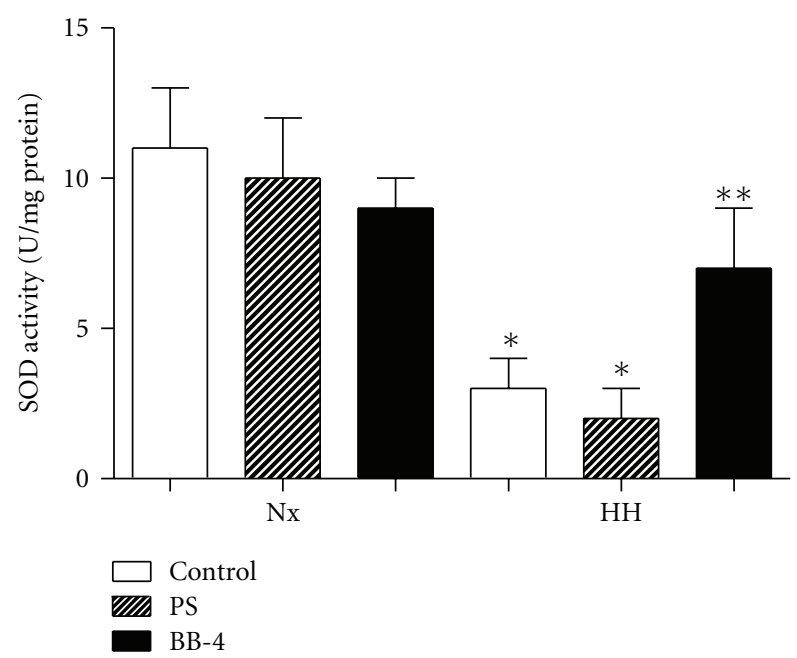

(c)

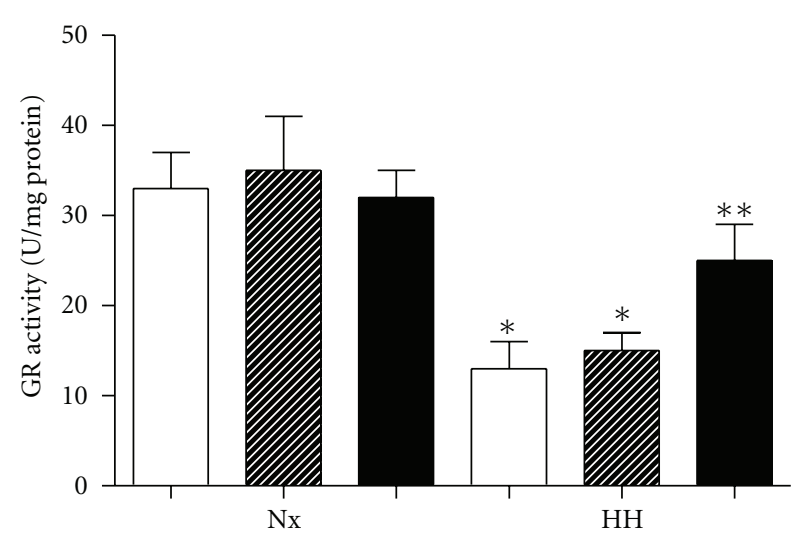

(b)
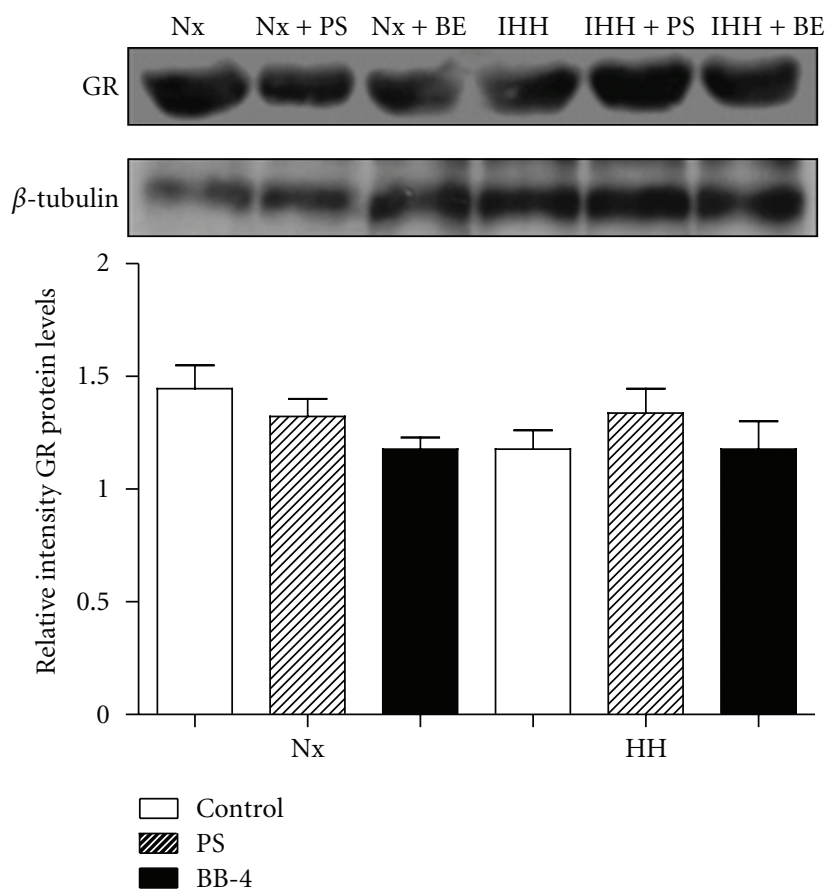

(d)

FIGURE 2: Effect of intermittent hypobaric hypoxia and BB-4 on (a) lipid peroxidation (TBARS) (nmol/mg protein), (b) glutathione reductase (GR) activity (U/mg protein), (c) superoxide dismutase (SOD) activity, and (d) glutathione reductase protein expression. Rats were submitted to intermittent hypobaric hypoxia $(\mathrm{IHH})$ or normobaric $(\mathrm{Nx})$ conditions, with or without treatment of blueberry extract (BB-4). PS: rats treated with physiological $\mathrm{NaCl}$ solution. Experiment: 32 -day treatment. Bars indicate the mean $\pm \mathrm{SD}(n=5)$. ${ }^{*} P<0.05$ ( $\mathrm{HH}$ versus $\mathrm{Nx}$ control); ${ }^{* *} \mathrm{P}<0.05$ ( $\mathrm{HH}+\mathrm{BB}-4$ versus $\mathrm{HH}$ control).

hypobaric hypoxia and treated with blueberry extract (BB4) showed a significant decrease in lipid peroxidation in rat testis reaching levels similar to normoxic condition. On the other hand, blueberry extract prevented the effects of hypobaric hypoxia on testicular mass, arrest of spermatogenesis, and apoptosis. The effect of blueberry extract on spermatogenic cells can be attributed to a possible passage through the hematotesticular barrier and thus protecting this tissue from oxidative stress generated by hypobaric hypoxia. Our results indicated that there were no changes in GR expression. However, the enzyme activity was significantly restored in animals subjected to hypobaric hypoxia and treated with blueberry extract suggesting that these compounds could activate the powerful endogenous antioxidant defenses by chemically reducing oxidized glutathione [33].

Hypobaric hypoxia caused a significant loss in body weight. The effect of hypoxia on body weight has been described by various authors who have indicated that mammals exposed to different hypoxia exposure experienced weight loss, dehydration, fat loss, and muscular mass reduction [34-36]. The blueberry extract did not have a protective effect on body weight. Our data suggested that blueberry 


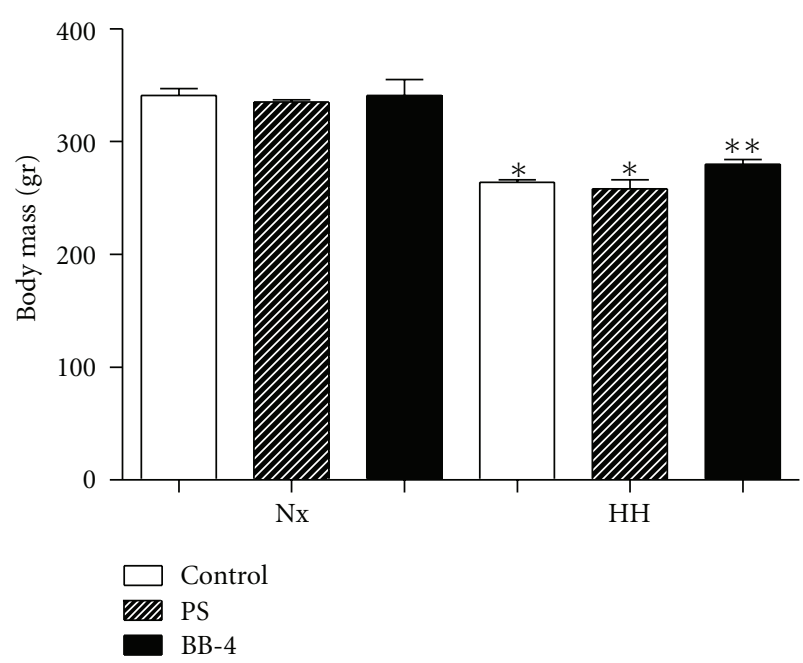

(a)

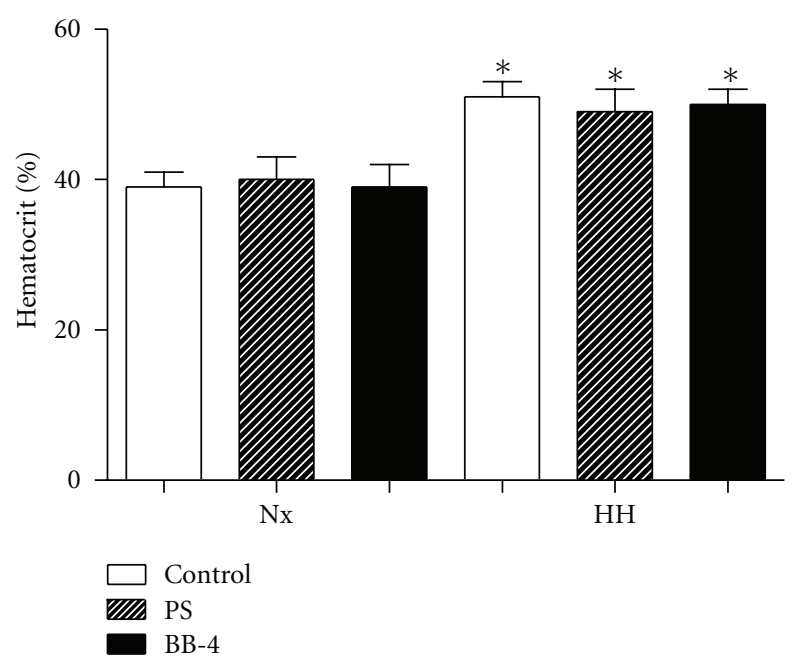

(b)

FIGURE 3: Effect of intermittent hypobaric hypoxia and BB-4 on (a) body weight and (b) hematocrit (\%). Rats were submitted to intermittent hypobaric hypoxia $(\mathrm{IHH})$ or normobaric $(\mathrm{Nx})$ conditions, with or without treatment of blueberry extract (BB-4). PS: rats treated with physiological $\mathrm{NaCl}$ solution. Experiment: 32 -day treatment. Bars indicate the mean $\pm \mathrm{SD}(n=5)$. ${ }^{*} P<0.05$ (HH versus $\mathrm{Nx}$ control); ${ }^{* *} \mathrm{P}<0.05$ (HH+BB-4 versus $\mathrm{HH}$ control).

extract may blunt the oxidative stress induced by hypobaric hypoxia as showed by the decreased lipid peroxidation and the increased glutathione reductase and superoxide dismutase activities with BB-4 compared to control. The dose of BB-4 did not affect polycythemia, a characteristic of exposure to hypobaric hypoxia as a compensatory mechanism to the drop in arterial $\mathrm{PO}_{2}$. A significant increase in erythrocytes was observed in animals exposed to intermittent hypobaric hypoxia, which indicated that BB-E had no effect on erythropoietin; therefore, it did not affect one of the mechanisms of acclimatization to high altitudes [37].

These results corroborate previous studies demonstrating the beneficial effects of polyphenols present in natural and enriched foods [18]. Blueberry extract presented a protective effect against oxidative stress induced by hypobaric hypoxia by recovering glutathione reductase and superoxide dismutase activities. It can be concluded that extracts of natural origin can be effective in the prevention of oxidative stress induced by hypobaric hypoxia, and it opens the possibility of generating additional health benefits to people who live under conditions of oxidative stress.

\section{Acknowledgments}

The technical assistance of Angela Gonzalez is greatly appreciated. The authors are sincerely thankful for support provided by DIUFRO Grant 2011-DI11-6001 (J. G. Farías), DIUFRO Grant 2012-DI12-5005 (J. G. Farías), FONDECYT Grant no. 1120006 (G. M. Calaf and J. G. Farías), and Convenio de Desempeño Universidad de Tarapacá-MINEDUC, Chile (G. M. Calaf).

\section{References}

[1] F. Giampieri, S. Tulipani, J. M. Alvarez-Suarez, J. L. Quiles, B. Mezzetti, and M. Battino, "The strawberry: composition, nutritional quality, and impact on human health," Nutrition, vol. 28, no. 1, pp. 9-19, 2012.

[2] J. S. Bland, "Phytonutrition, phytotherapy, and phytopharmacology," Alternative Therapies in Health and Medicine, vol. 2, no. 6, pp. 73-76, 1996.

[3] J. M. Kong, L. S. Chia, N. K. Goh, T. F. Chia, and R. Brouillard, "Analysis and biological activities of anthocyanins," Phytochemistry, vol. 64, no. 5, pp. 923-933, 2003.

[4] S. Zafra-Stone, T. Yasmin, M. Bagchi, A. Chatterjee, J. A. Vinson, and D. Bagchi, "Berry anthocyanins as novel antioxidants in human health and disease prevention," Molecular Nutrition and Food Research, vol. 51, no. 6, pp. 675-683, 2007.

[5] P. Rani, K. M. Unni, and J. Karthikeyan, "Evaluation of antioxidant properties of berries," Indian Journal of Clinical Biochemistry, vol. 19, no. 2, pp. 103-110, 2004.

[6] D. Bagchi, C. K. Sen, M. Bagchi, and M. Atalay, "Antiangiogenic, antioxidant, and anticarcinogenic properties of a novel anthocyanin-rich berry extract formula," Biokhimiya, vol. 69, no. 1, pp. 95-102, 2004.

[7] S. J. Duthie, A. M. Jenkinson, A. Crozier et al., "The effects of cranberry juice consumption on antioxidant status and biomarkers relating to heart disease and cancer in healthy human volunteers," European Journal of Nutrition, vol. 45, no. 2, pp. 113-122, 2006.

[8] J. O. Moskaug, H. Carlsen, M. C. Myhrstad, and R. Blomhoff, "Polyphenols and glutathione synthesis regulation," The American Journal of Clinical Nutrition, vol. 81, no. 1, pp. 277S283S, 2005.

[9] S. M. Khan and G. Kour, "Subacute oral toxicity of chlorpyriphos and protective effect of green tea extract," Pesticide Biochemistry and Physiology, vol. 89, no. 2, pp. 118-123, 2007.

[10] M. E. Anderson and A. Meister, "Transport and direct utilization of $\gamma$-glutamylcyst(e)ine for glutathione synthesis," 
Proceedings of the National Academy of Sciences of the United States of America, vol. 80, no. 3, pp. 707-711, 1983.

[11] J. G. Farias, M. Puebla, A. Acevedo et al., "Oxidative stress in rat testis and epididymis under intermittent hypobaric hypoxia: protective role of ascorbate supplementation," Journal of Andrology, vol. 31, no. 3, pp. 314-321, 2010.

[12] J. G. Farías, E. Bustos-Obregón, and J. G. Reyes, "Increase in testicular temperature and vascularization induced by hypobaric hypoxia in rats," Journal of Andrology, vol. 26, no. 6, pp. 693-697, 2005.

[13] J. G. Farias, E. Bustos-Obregón, R. Orellana, J. L. Bucarey, E. Quiroz, and J. G. Reyes, "Effects of chronic hypobaric hypoxia on testis histology and round spermatid oxidative metabolism," Andrologia, vol. 37, no. 1, pp. 47-52, 2005.

[14] J. G. Farias, A. B. Zepeda, and G. M. Calaf, "Melatonin protects the heart, lungs and kidneys from oxidative stress under intermittent hypobaric hypoxia in rats," Biological Research, vol. 45, pp. 81-85, 2012.

[15] G. H. Fong and K. Takeda, "Role and regulation of prolyl hydroxylase domain proteins," Cell Death and Differentiation, vol. 15 , no. 4, pp. 635-641, 2008.

[16] E. Bustos-Obregón, R. Castro-Sánchez, B. Ramos-González, and L. Torres-Díaz, "Rat spermatogenesis damage in intermittent hypobaric hypoxia and the protective role of melatonin. II: Testicular parameters," International Journal of Morphology, vol. 28, no. 2, pp. 537-547, 2010.

[17] R. Hartley, R. Castro-Sánchez, B. Ramos-Gonzalez, and E. Bustos-Obregón, "Rat spermatogenesis damage in intermittent hypobaric hypoxia and the protective role of melatonin. I Cauda epididymal spermatozoa," International Journal of Morphology, vol. 27, no. 4, pp. 1275-1284, 2009.

[18] J. Fuentealba, A. J. Dibarrart, M. C. Fuentes-Fuentes et al., "Synaptic failure and adenosine triphosphate imbalance induced by amyloid- $\beta$ aggregates are prevented by blueberry-enriched polyphenols extract," Journal of Neuroscience Research, vol. 89, no. 9, pp. 1499-1508, 2011.

[19] D. H. Malin, D. R. Lee, P. Goyarzu et al., "Short-term blueberry-enriched diet prevents and reverses object recognition memory loss in aging rats," Nutrition, vol. 27, no. 3, pp. 338-342, 2011.

[20] C. Rendeiro, D. Vauzour, R. J. Kean et al., "Blueberry supplementation induces spatial memory improvements and regionspecific regulation of hippocampal BDNF mRNA expression in young rats," Psychopharmacology, vol. 223, no. 3, pp. 319330, 2012.

[21] M. G. Alves, P. F. Oliveira, S. Socorro, and P. I. Moreira, "Impact of diabetes in blood-testis and blood-brain barriers: resemblances and differences," Current Diabetes Reviews, vol. 8, no. 6, pp. 401-412, 2012.

[22] M. M. Bradford, "A rapid and sensitive method for the quantitation of microgram quantities of protein utilizing the principle of protein dye binding," Analytical Biochemistry, vol. 72, no. 1-2, pp. 248-254, 1976.

[23] V. Lesauskaite, M. C. Epistolato, L. Ivanoviene, and P. Tanganelli, "Apoptosis of cardiomyocytes in explanted and transplanted hearts: comparison of results from in situ TUNEL, ISEL, and ISOL reactions," American Journal of Clinical Pathology, vol. 121, no. 1, pp. 108-116, 2004.

[24] B. Weiss, A. Jacquemin-Sablon, T. R. Live, G. C. Fareed, and C. C. Richardson, "Enzymatic breakage and joining of deoxyribonucleic acid. VI. Further purification and properties of polynucleotide ligase from Escherichia coli infected with bacteriophage T4," Journal of Biological Chemistry, vol. 243, no. 17 , pp. $4543-4555,1968$.
[25] H. H. Draper and M. Hadley, "Malondialdehyde determination as index of lipid peroxidation," Methods in Enzymology, vol. 186, pp. 421-431, 1990.

[26] V. M. Rodríguez, L. M. Del Razo, J. H. Limón-Pacheco et al., "Glutathione reductase inhibition and methylated arsenic distribution in Cd1 mice brain and liver," Toxicological Sciences, vol. 84, no. 1, pp. 157-166, 2005.

[27] S. Marklund and G. Marklund, "Involvement of the superoxide anion radical in the autoxidation of pyrogallol and a convenient assay for superoxide dismutase," European Journal of Biochemistry, vol. 47, no. 3, pp. 469-474, 1974.

[28] R. J. Aitken and S. D. Roman, "Antioxidant systems and oxidative stress in the testes," Oxidative Medicine and Cellular Longevity, vol. 1, no. 1, pp. 15-24, 2008.

[29] E. Demir, B. Kaya, C. Soriano, A. Creus, and R. Marcos, "Genotoxic analysis of four lipid-peroxidation products in the mouse lymphoma assay," Mutation Research, vol. 726, no. 2, pp. 98-103, 2011.

[30] R. L. Prior and G. Cao, "Analysis of botanicals and dietary supplements for antioxidant capacity: a review," Journal of AOAC International, vol. 83, no. 4, pp. 950-956, 2000.

[31] Y. Kalender, S. Kaya, D. Durak, F. G. Uzun, and F. Demir, "Protective effects of catechin and quercetin on antioxidant status, lipid peroxidation and testis-histoarchitecture induced by chlorpyrifos in male rats," Environmental Toxicology and Pharmacology, vol. 33, no. 2, pp. 141-148, 2012.

[32] X. Wu, J. Kang, C. Xie et al., "Dietary blueberries attenuate atherosclerosis in apolipoprotein E-deficient mice by upregulating antioxidant enzyme expression," Journal of Nutrition, vol. 140, no. 9, pp. 1628-1632, 2010.

[33] J. A. Joseph, N. A. Denisova, D. Bielinski, D. R. Fisher, and B. Shukitt-Hale, "Oxidative stress protection and vulnerability in aging: putative nutritional implications for intervention," Mechanisms of Ageing and Development, vol. 116, no. 2-3, pp. 141-153, 2000.

[34] E. J. Clegg, "Weight changes in different organs of the mouse at two levels of reduced atmospheric pressure," Journal of Applied Physiology, vol. 30, no. 5, pp. 764-767, 1971.

[35] D. Garayar and R. Guerra-García, "Auxología, endocrinología y morfometría del cobayo (Cavia aperea) a nivel del mar y en la altura," Acta Andina, vol. 2, pp. 101-108, 1992.

[36] J. G. Farias, E. Bustos-Obregón, P. J. Tapia et al., "Time course of endocrine changes in the hypophysis-gonad axis induced by hypobaric hypoxia in male rats," Journal of Reproduction and Development, vol. 54, no. 1, pp. 18-21, 2008.

[37] K. U. Eckardt, U. Boutellier, A. Kurtz, M. Schopen, E. A. Koller, and C. Bauer, "Rate of erythropoietin formation in humans in response to acute hypobaric hypoxia," Journal of Applied Physiology, vol. 66, no. 4, pp. 1785-1788, 1989. 


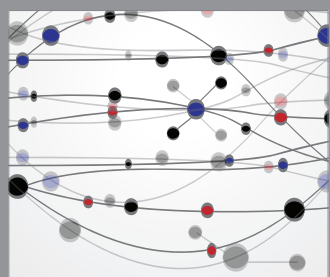

The Scientific World Journal
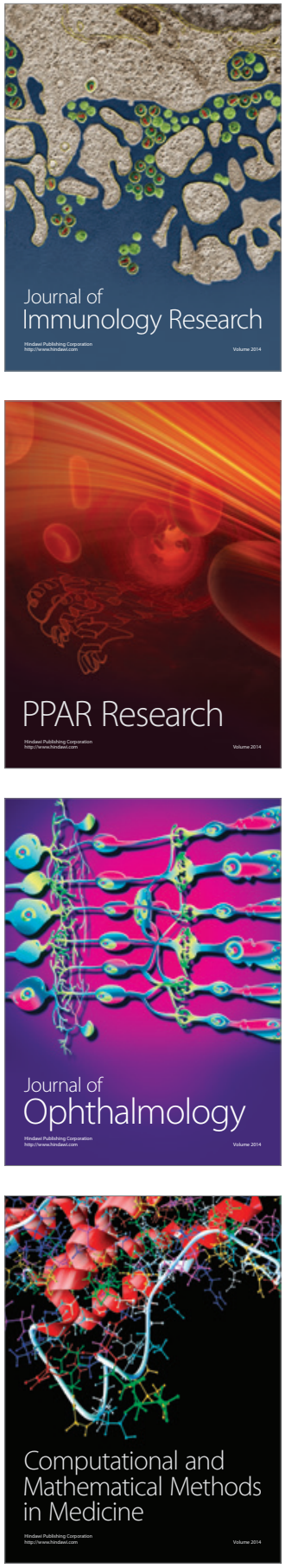

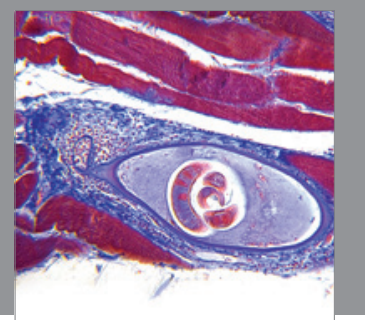

Gastroenterology

Research and Practice
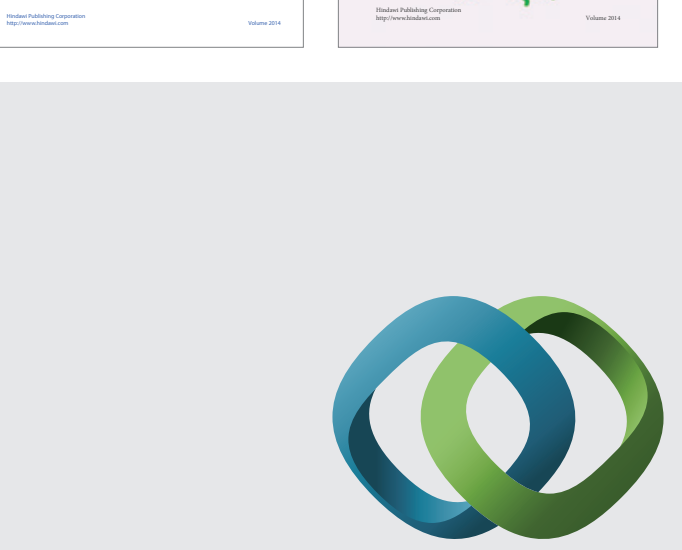

\section{Hindawi}

Submit your manuscripts at

http://www.hindawi.com
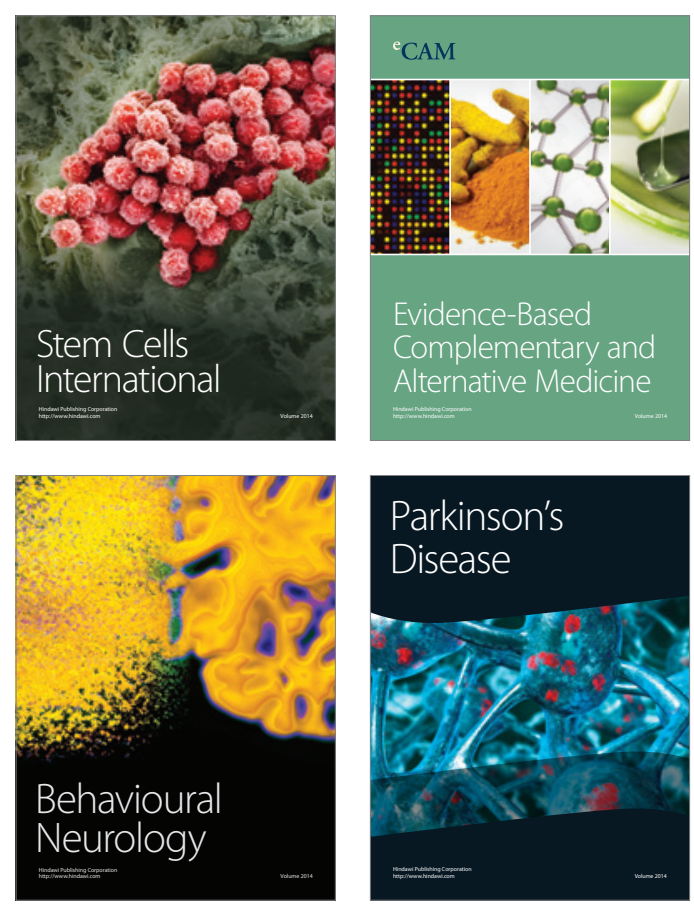

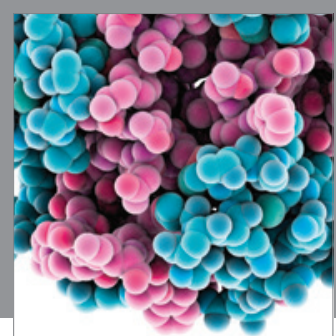

Journal of
Diabetes Research

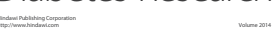

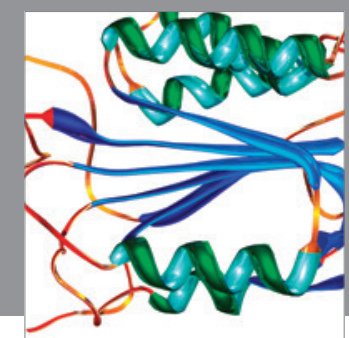

Disease Markers
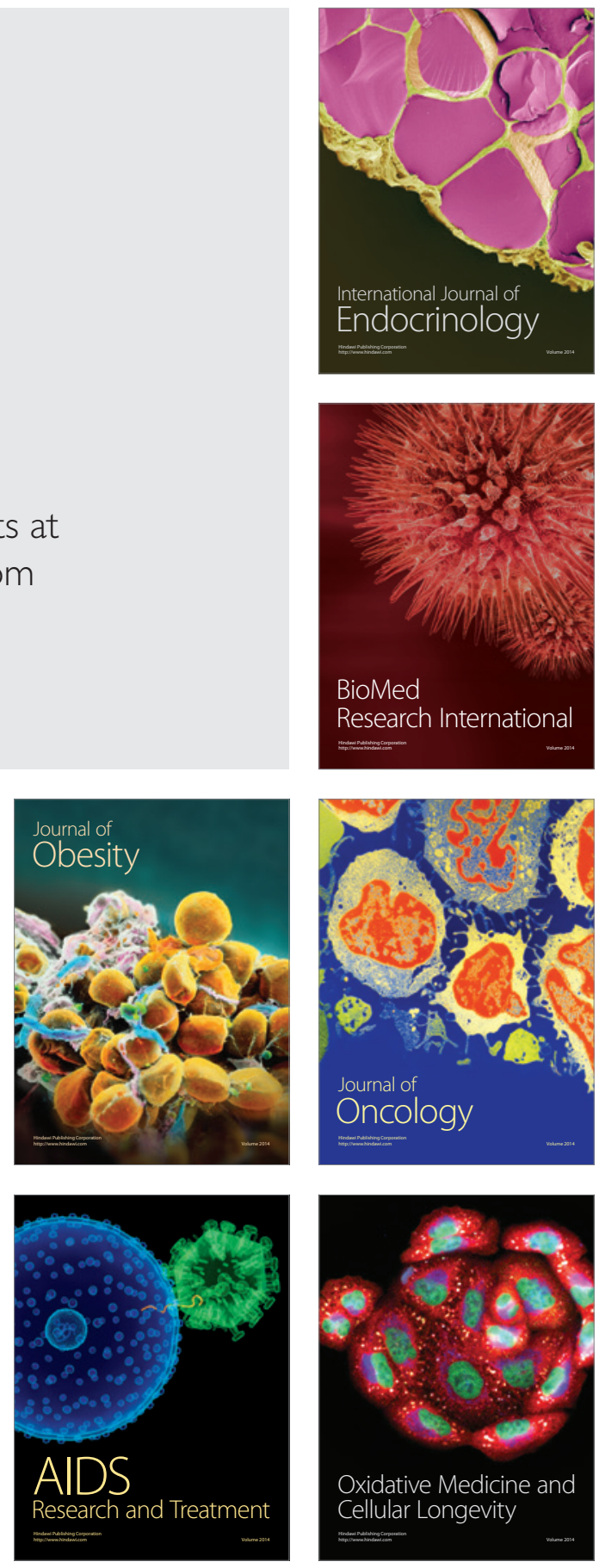